\title{
The benefits of restraint: a pivotal role for IL-13 in hepatic glucose homeostasis
}

\author{
Mariana Verdelho Machado, ${ }^{1}$ Yiping Yang, ${ }^{2}$ and Anna Mae Diehl' \\ ${ }^{1}$ Division of Gastroenterology and 2Division of Oncology, Duke University School of Medicine, Durham, North Carolina, USA.
}

\begin{abstract}
In response to feeding, insulin promotes the uptake of sugar in peripheral tissues and suppresses the production of sugar, a process called gluconeogenesis, in the liver. Recent research has shown that chronic inflammation promotes insulin resistance, and in turn, chronically high glucose levels can drive inflammation. In this issue of the JCI, Stanya et al. investigate the connection between inflammation and glucose homeostasis by analyzing the effect of the antiinflammatory cytokine IL-13. Their results suggest that IL-13 plays an unexpected role in the regulation of glucose homeostasis by modulating gluconeogenesis and may be a useful therapeutic target for treatment of diabetes and metabolic syndrome.
\end{abstract}

Obesity and deregulated glucose metabolism are the pandemic of the twenty-first century. One-fourth of Americans have metabolic syndrome (1), a combination of insulin resistance, obesity, dyslipidemia, and hypertension that in the liver manifests as nonalcoholic fatty liver disease (NAFLD). The liver is believed to play a pivotal role in the pathogenesis of metabolic syndrome because postprandial hyperglycemia is one of the earliest manifestations of this condition. Unlike healthy individuals, those with metabolic syndrome fail to downregulate hepatic gluconeogenesis appropriately after eating. The resultant hyperglycemia stimulates the pancreas to increase insulin secretion. Recurrent hyperinsulinemia results in insulin resistance, and in turn, the excessive demand for insulin production can ultimately overwhelm pancreatic $\beta$ cell capacity, leading to fasting hyperglycemia, i.e., type 2 diabetes mellitus (T2DM). Data from the Centers for Disease Control show that there has been a $160 \%$ increase in the prevalence of T2DM in the past 20 years, such that T2DM now afflicts about $9 \%$ of the US population (i.e., more than 20 million Americans; ref. 2). Despite tremendous efforts by the scientific community, the mechanisms that deregulate hepatic glucose metabolism in metabolic syndrome are not fully understood.

Conflict of interest: The authors have declared that no conflict of interest exists.

Citation for this article: J Clin Invest. 2013; 123(1):115-117. doi:10.1172/JCI67238.
Obesity is a major risk factor for metabolic syndrome; thus, considerable effort has focused on identifying mechanisms by which adiposity might disrupt hepatic glucose homeostasis. A seminal paper by Hotamisligil et al. revealed an association among obesity, increased adipose-derived TNF- $\alpha$, and insulin resistance (3). Subsequently, it was shown that obese mice lacking TNF- $\alpha$ had improved insulin sensitivity and glucose homeostasis and that exogenous administration of TNF- $\alpha$ led to insulin resistance. In obese humans, TNF- $\alpha$ is overexpressed in muscle and adipose tissue, and agents that are used to inhibit TNF- $\alpha$ and other proinflammatory (also known as Th1) cytokines in patients with chronic inflammatory diseases improve insulin sensitivity (4). Several proinflammatory cytokines, including TNF- $\alpha$, activate JNK, which inhibits insulin receptor signaling through serine phosphorylation and inactivation of its downstream target, IRS-1. A new concept emerged of crosstalk between the immune system and metabolism, buttressed by the concept that integrating nutrient and pathogen sensing with immune responses is desirable from an evolutionary perspective, since mounting an inflammatory response is metabolically expensive (5).

\section{A new role for IL-13}

The study by Stanya et al. in this issue advances our understanding of the intricate relationships between the immune system and metabolism (6). By studying two different strains of mice genetically deficient in IL-13, a key cytokine in the antiinflammatory (also known as Th2 or M2) arm of the immune system, the authors demonstrated that IL-13 is required for normal postprandial suppression of hepatic glucose production. In mice on the C57BL/6 genetic background, which is skewed toward proinflammatory responses, IL-13 deficiency resulted in deregulation of postprandial hepatic glucose metabolism at a young age in chow-fed animals. This led to postprandial hyperglycemia, insulin resistance, decreased oxygen consumption, weight gain, and increased levels of triglyceride in blood and liver, i.e., emergence of metabolic syndrome. Notably, deregulated hepatic glucose homeostasis occurred without an obvious increase in proinflammatory cytokines or alterations in liver or adipose depot macrophages. Moreover, the defective liver glucose metabolism antedated the slight increase in age-related adiposity that accompanied IL-13 deficiency by several months. When IL-13 was knocked out in BALB/c mice, which are skewed toward antiinflammatory responses and are less prone to metabolic disease, a high-fat diet challenge was required to elicit deregulated hepatic glucose homeostasis. In addition, IL-13 deficiency led to decreased glucose uptake in white adipose tissue.

The authors went on to demonstrate that the effects of IL-13 on hepatic glucose production are mediated by phosphorylation of STAT3 (p-STAT3; ref. 6). Previously, IL-13 receptor $\alpha$ (IL-13R $\alpha$ ) had been linked to p-STAT3 accumulation in immune cells (7), and hepatocytes were known to express IL-13R $\alpha$ (8). In the current study, when isolated hepatocytes were treated with recombinant IL-13, p-STAT3 accumulated and suppressed gluconeogenic gene expression, resulting in reduced hepatocyte glucose production. The actions of IL-13 were abrogated in STAT3-deficient hepatocytes, but unaltered in STAT6-deficient cells (6). The authors' results therefore revealed that IL-13 is one of the master regulators of glucose metabolism, acting by directly inhibiting the transcription of hepatic genes that encode key gluconeogenic enzymes, such as phosphoenolpyruvate carboxykinase (PEPCK) 
and glucose-6-phosphatase (G6P). In mice, this action appeared to be relevant mainly in the postprandial period, when a sudden increase in the availability of glucose could lead to a tremendous increase in glycemia.

Although the murine data predict that healthy humans will also increase hepatic production of IL-13 after eating, this has not yet been directly examined. Measurement of serum cytokine levels in a small number of patients demonstrated higher serum levels of both pro- and antiinflammatory cytokines (including IL-13) in subjects with metabolic syndrome, prompting speculation that the antiinflammatory cytokines might have been induced to counteract excessive proinflammatory signaling (9). Overproduction of IL-13 might be harmful to the liver, however, because IL-13 is known to directly activate hepatic stellate cells to a fibrogenic phenotype (10). Moreover, strong positive correlations between IL-13 and advanced stages of NAFLD - specifically, steatohepatitis and liver fibrosis - have been reported $(11,12)$. Consistent with that concept, rats with diet-induced steatohepatitis that were treated with an IL-13 receptor-directed cytotoxin showed decreased liver injury and fibrosis (11). These findings underscore the complexity of immune system/metabolic interactions and emphasize the importance of mechanisms that function to balance proand antiinflammatory signaling.

\section{Identifying the source}

The liver harbors many types of cells that are capable of producing cytokines. To uncover the cellular sources of IL-13 in the liver, Stanya et al. harvested mononuclear cells from the livers of mice with a GFP knockin in the IL-13 locus, and used FACS to identify IL-13-positive populations. In both fed and fasted conditions, NKT cells proved to be the main source of hepatic IL-13 (6). This discovery is notable in light of some of the unique properties of NKT cells as well as previous research that has already linked this type of immune cell with insulin resistance.

NKT cells - a distinct lineage of T lymphocytes - share features with NK cells, acting as a bridge between innate and adaptive immunity. Unlike conventional T cells, NKT cells recognize exogenous and endogenous glycolipids presented by the nonpolymorphic class I MHC-like molecule CD1d. Interestingly, although NKT cells are not abundant in peripheral blood, they are highly enriched in adipose tissue and liver. NKT cells represent $30 \%$ of intrahepatic lymphocytes in mice and $10 \%$ in humans
(13). Interestingly, the highest expression of CD1d occurs in hepatocytes and adipocytes, rather than in classical antigen-presenting cells, suggesting a role for NKT cells in metabolism regulation (14). Additionally, CD1d expression is inducible by PPAR $\gamma$, a major regulator of lipid metabolism that is activated by fatty acids and other lipids (15).

\section{A complicated relationship}

Earlier research has linked NKT cells with obesity and glucose metabolism. For example, in humans, the number of adipose tissue resident NKT cells inversely correlates with body mass index, fasting glucose, and insulin resistance (16). Consistent with that observation, a decrease in intrahepatic NKT resident cells been documented in humans with hepatic steatosis (17). Studies in mice with either genetic or diet-induced obesity and hepatic steatosis have also consistently shown a decrease in hepatic and adipose CD4-positive NKT cells $(14,18-23)$. Several mechanisms appear to mediate this reduction in hepatic NKT cells, including defective hepatic homing of NKT cells (18), decreased CD1d presentation by hepatocytes and antigen-presenting cells due to lipid-induced endoplasmic reticulum stress $(20,21)$, and increased NKT apoptosis mediated by local increases in IL-12 (17-19). Such NKT cell depletion may contribute to metabolic deregulation, because mice defective for NKT cells (e.g., CD1d- or Ja18-deficient mice) are more vulnerable to diet-induced obesity, hepatic steatosis, and insulin resistance than are wild-type mice $(14,23)$. A causal role for liver NKT cell depletion in hepatic steatosis and insulin resistance is further supported by evidence that stimulation of NKT cells with glucocerebroside, or adoptive transfer of NKT cells, improves liver steatosis and glucose tolerance in genetically obese $o b / o b$ mice $(24,25)$. Similarly, activation of NKT cells with $\alpha$-galactosylceramide was shown to improve glucose homeostasis in wild-type mice with diet-induced obesity (16). That study demonstrated that activating NKT cells in adipose depots polarized local macrophage populations toward an antiinflammatory phenotype, and linked improvements in adipose insulin sensitivity to enhanced production of IL-4 and the resultant induction of STAT6. Although optimal control of glucose utilization in adipose depots requires regulatory input from NKT cells, in humans (as in mice), excessive hepatic accumulation of NKT cells increases local production of fibrogenic factors, including IL-13. Thus, it is not sur- prising that excessive accumulation of NKT cells has been demonstrated in human livers with advanced stages of fatty damage, such as steatohepatitis and cirrhosis $(26,27)$. The aggregate data therefore demonstrated that adverse outcomes result from either hepatic depletion of NKT cells or excessive hepatic accumulation of NKT cells.

\section{Unresolved questions}

The study by Stanya et al. advances understanding about NKT cell-related mechanisms that enhance metabolic control and suggest that NKT cells in different insulin target tissues, such as fat and liver, normally collaborate to orchestrate coordinated responses to feeding (6). However, although NKT cells are a main source of IL-13 in the liver, and it is reasonable to presume that feeding-related changes in hepatic lipid content might affect their activation, NKT cell numbers per se are not altered by feeding (5). This suggests that other types of IL-13-producing cells might also be involved in postprandial regulation of hepatic glucose output. Indeed, Stanya et al. identified a population of hepatic non$\mathrm{T}$, non-NKT cells that upregulates its production of IL-13 in response to a meal (6). The nature of this population, and the biological significance of the IL-13 it produces, remain to be determined. Further research is also needed to delineate the precise contribution of different IL-13-producing cells in regulating glucose metabolism.

\section{Conclusions}

There is growing evidence that glucose homeostasis in metabolically active tissues, such as fat and liver, is regulated by immune cells that are embedded within them. This process involves cytokine-mediated crosstalk among NKT cells and neighboring cells, including macrophages and parenchymal cells. Moreover, recent data show that antiinflammatory cytokines, such as IL-13 and IL-4, are no less important for glucose homeostasis than are proinflammatory cytokines, such as TNF- $\alpha$, that have long been credited as metabolic regulators. Indeed, the present work by Stanya et al. (6) suggests a completely novel paradigm for the pathogenesis of insulin resistance and T2DM: that they may result from a primary defect in the antiinflammatory arm of the immune system. This defect prevents sufficient hepatic production of IL-13, an antiinflammatory cytokine that is directly required to suppress hepatic glucose production after feeding. 
Given that the liver receives blood draining into its sinusoids from the intestines and mesenteric fat, and that NKT cells patrol liver sinusoids, this discovery raises the intriguing possibility that immune-mediated mechanisms that suppress postprandial glucose production are inherently coupled to those that dampen hepatic immune responses to the gut-derived factors that have been implicated in inflammasome activation and deregulation of neurohumoral mechanisms that control feeding behavior and energy homeostasis. This insight, in turn, may have broad implications for the development of novel strategies to control obesity, insulin resistance, and T2DM.

Address correspondence to: Anna Mae Diehl, Division of Gastroenterology, Snyderman Building, Suite 1073, Duke University, Durham, North Carolina 27710, USA. Phone: 919.684.4173; Fax: 919.684.4183; E-mail: annamae.diehl@dm.duke.edu.

1. Meigs JB. Epidemiology of the insulin resistance syndrome. Curr Diab Rep. 2003;3(1):73-79.

2. Centers for Disease Control and Prevention. Diabetes Report Card 2012. Atlanta, Georgia, USA: Centers for Disease Control and Prevention, US Department of Health and Human Services; 2012.

3. Hotamisligil GS, Shargill NS, Spiegelman BM Adipose expression of tumor necrosis factor-alpha: direct role in obesity-linked insulin resistance. Science. 1993;259(5091):87-91.

4. Hotamisligil GS. Inflammation and metabolic disorders. Nature. 2006;444(7121):860-867.

5. Hotamisligil GS, Erbay E. 2008. Nutrient sensing and inflammation in metabolic diseases. Nat Rev Immunol. 2008;8(12):923-934.

6 . Stanya KJ, et al. Direct control of hepatic glucose production by interleukin-13 in mice. J Clin Invest. 2013;123(1):261-271

7. Roy B, Bhattacharjee A, Xu B, Ford D, Maizel $\mathrm{AL}$, Cathcart MK. IL-13 signal transduction in human monocytes: phosphorylation of receptor components, association with Jaks, and phosphorylation/activation of Stats. J Lenkoc Biol. 2003 ; 72(3):580-589.

8. Akaiwa $\mathrm{M}$, et al. Localization of human interleukin 13 receptor in non-haematopoietic cells. Cytokine. 2001;13(2):75-84

9. Surendar J, Mohan V, Rao MM, Babu S, Aravindhan V. Increased levels of both Th1 and Th2 cytokines in subjects with metabolic syndrome (CURES-103). Diabetes Technol Ther. 2001;13(4):477-482.

10. Sugimoto R, et al. 2005. Effect of IL-4 and IL-13 on collagen production in cultured LI90 human hepatic stellate cells. Liver Int. 2005;25(2):420-428.

11. Shimamura T, Fujisawa T, Husain SR, Kioi M, Nakajima A, Puri RK. 2008. Novel role of IL-13 in fibrosis induced by nonalcoholic steatohepatitis and its amelioration by IL-13R-directed cytotoxin in a rat model. J Immunol. 2008;181(7):4656-4665.

12. Weng HL, et al. 2009. The etiology of liver damage imparts cytokines transforming growth factor beta 1 or interleukin-13 as driving forces in fibrogenesis. Hepatology. 2009;50(1):230-243.

13. Gao B, Jeong WI, Tian Z. 2008. Liver: An organ with predominant innate immunity. Hepatology. 2008; 47(2):729-736.

14. Kotas ME, et al. Impact of CD1d deficiency on metabolism. PLoS One. 2012;6(9):e25478.

15. Szatmari I, et al. PPARgamma controls CD1d expression by turning on retinoic acid synthesis in developing human dendritic cells. J Exp Med. 2006; 203(10):2351-2362.

16. Ji Y, et al. Activation of natural killer T cells promotes M2 Macrophage polarization in adipose tissue and improves systemic glucose tolerance via interleukin-4 (IL-4)/STAT6 protein signaling axis in obesity. J Biol Chem. 2012;287(17):13561-13571.
17. Kremer M, et al. Kupffer cell and interleukin-12-dependent loss of natural killer T cells in hepatosteatosis. Hepatology. 2010;51(1):130-141.

18. Guebre-Xabier M, Yang S, Lin HZ, Schwenk R, Krzych U, Diehl AM. Altered hepatic lymphocyte subpopulations in obesity-related murine fatty livers: potential mechanism for sensitization to liver damage. Hepatology. 2000;31(3):633-640.

19. Li Z, Soloski MJ, Diehl AM. Dietary factors alter hepatic innate immune system in mice with nonalcoholic fatty liver disease. Hepatology. 2005; 42(4):880-885.

20. Yang L, Jhaveri R, Huang J, Qi Y, Diehl AM. Endoplasmic reticulum stress, hepatocyte CD1d and NKT cell abnormalities in murine fatty livers. $L a b$ Invest. 2007;87(9):927-937.

21. Miyazaki Y, et al. Effect of high fat diet on NKT cell function and NKT cell-mediated regulation of Th1 responses. Scand J Immunol. 2008;67(3):230-237.

22. Mantell BS, Stefanovic-Racic M, Yang X, Dedousis N, Sipula IJ, O’Doherty RM. Mice lacking NKT cells but with a complete complement of CD8+ T-cells are not protected against the metabolic abnormalities of diet-induced obesity. PLoS One. 2011; 6(6):e19831.

23. Lynch L, et al. Adipose tissue invariant NKT cells protect against diet-induced obesity and metabolic disorder through regulatory cytokine production. Immunity. 2012;37(3):574-587.

24. Margalit M, et al. Glucocerebroside ameliorates the metabolic syndrome in $\mathrm{OB} / \mathrm{OB}$ mice. J Pharmacol Exp Ther. 2006;319(1):105-110.

25. Elinav E, et al. Adoptive transfer of regulatory NKT lymphocytes ameliorates non-alcoholic steatohepatitis and glucose intolerance in ob/ob mice and is associated with intrahepatic CD8 trapping. J Pathol. 2006;209(1):121-128

26. Syn WK, et al. Accumulation of natural killer T cells in progressive nonalcoholic fatty liver disease. Hepatology. 2010;51(6):1998-2007.

27. Syn WK, et al. NKT cells stimulate hedgehog and osteopontin production to drive fibrogenesis in nonalcoholic fatty liver disease. Gut. 2012; 61(9):1323-1329.

\title{
A heartfelt response: new thyroid hormone- sensitive neurons in the hypothalamus
}

\author{
Douglas Forrest ${ }^{1}$ and Jürgen Wess ${ }^{2}$
}

${ }^{1}$ Nuclear Receptor Biology Section, Laboratory of Endocrinology and Receptor Biology, and 2Molecular Signaling Section, Laboratory of Bioorganic Chemistry, National Institute of Diabetes and Digestive and Kidney Diseases (NIDDK), Bethesda, Maryland, USA.

\begin{abstract}
Thyroid hormone is a well-known regulator of metabolic and cardiovascular functions, and signaling through thyroid receptors has differential effects on cells depending on the receptor isoform that they express. In this issue of the JCI, Mittag et al. provide evidence that thyroid hormone receptors are essential for the formation of a population of parvalbuminergic neurons in the anterior hypothalamus, linking, for the first time, impaired thyroid hormone signaling during development to cellular deficits in the hypothalamus. Since this newly discovered cell group is predicted to play a role in regulating cardiovascular function, these findings suggest that developmental hypothyroidism may be the cause of cardiovascular disorders later in life.
\end{abstract}

Conflict of interest: The authors have declared that no conflict of interest exists.

Citation for this article: J Clin Invest. 2013;

123(1):117-120. doi:10.1172/JCI67448.
Thyroid hormone plays a key role in regulating many developmental, metabolic, and cardiovascular processes $(1,2)$. Congenital hypothyroidism, which occurs in 1 in less than 3,000 births (3), and other thyroid gland disorders are associated with defects in the maturation and function of many tissues and organ systems. It has been a long-standing challenge to decipher the mechanisms by which thyroid hormone regulates such a wide range of cellular processes in so many different tissues. How does the same hormone stimulate the differentiation of an embryonic neuroblast but trigger an entirely different response in an adult liver cell? Thyroid hormone acts through the intracellular thyroid hormone receptor (TR), which belongs to the nuclear receptor family and acts as a ligand-regu- 